\title{
AN EFFICIENT DEMOSAICING APPROACH WITH A GLOBAL CONTROL OF CORRECTION STEPS
}

\author{
K.N. Plataniotis and R. Lukac \\ Bell Canada Multimedia Laboratory, The Edward S. Rogers Sr. Department of ECE, \\ University of Toronto, 10 King's College Road, Toronto, Canada \\ kostas@dsp.utoronto.ca,lukacr@ieee.org
}

\begin{abstract}
The paper introduces a new color filter array (CFA) interpolation method for digital still cameras. The proposed interpolation scheme is able to overcome the limitations of existing CFA based image acquisition solutions, and restore color images without introducing false colors, edge blurring or visual artifacts. The method utilizes an edge-sensing mechanism, a color-difference model and a correction step. Since the employed correction process is useful in highly correlated images, however, in images with globally week correlation variations is counter productive, a global control of the correction step is introduced. Simulation studies indicate that the proposed method is computationally efficient and yields excellent performance, in terms of subjective and objective image quality measures, while outperforming state-of-the-art CFA interpolation methods.
\end{abstract}

\section{INTRODUCTION}

The commercial proliferation of single-sensor digital cameras and their use in novel applications such as wireless phones, sensor networks, pocket devices, surveillance, and automotive apparatus has increased the demand for new algorithmic and technical developments in the area of color array (CFA) interpolation. The Bayer pattern (Fig.1) [2], the most widely used CFA, provides an array or mosaic of Red $(\mathrm{R})$, Green (G) and Blue (B) colors in which only one color element is available at each spatial location. The Bayer pattern has $50 \%$ green positions arranged in a checkerboard format while alternative lines of red and blue positions make the rest of the pattern. By allocating more spatial samples to the Green plane, the Bayer pattern improves the perceived sharpness of the digital image since it is well-known that the human visual system (HVS) is more sensitive to luminance which is composed primarily of green light [9]. The missing color components are recovered from the adjacent Bayer data using the so-called CFA interpolation or demosaicing process [4],[7].

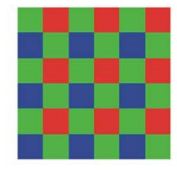

Fig. 1. Bayer color filter array.

\section{PROBLEM FORMULATION}

Let us consider, a $K_{1} \times K_{2}$ gray-scale image $z(i): Z^{2} \rightarrow Z$ representing a two-dimensional matrix of integer samples. In the Bayer CFA pattern, half of the pixels $z_{i} \in Z^{2}$, for $i=$ $1,2, \ldots, K_{1} K_{2}$, correspond to the $\mathrm{G}$ channel, whereas $\mathrm{R}$, $\mathrm{B}$ channels are assigned the other half of the pixels. Assuming that $p=1,2, \ldots, K_{1}$ and $q=1,2, \ldots, K_{2}$ denote the spatial position of the pixels in vertical (image rows) and horizontal (image columns) directions, gray-scale pixels $z_{i}$ can be transformed into the RGB vectors $\mathbf{x}_{i}=\left(x_{i 1}, x_{i 2}, x_{i 3}\right) \in$ $Z^{2}$, for $i=(p-1) K_{2}+q$, as follows:

$$
\mathbf{x}_{i}= \begin{cases}\left(z_{i}, 0,0\right) & \text { for } p \text { odd and } q \text { even } \\ \left(0,0, z_{i}\right) & \text { for } p \text { even and } q \text { odd } \\ \left(0, z_{i}, 0\right) & \text { otherwise }\end{cases}
$$

This transformation forms a $K_{1} \times K_{2}$ RGB image $\mathbf{x}(i)$ : $Z^{2} \rightarrow Z^{3}$ representing a two-dimensional matrix of threecomponent samples. Note that the color vectors $\mathbf{x}_{i}$ relate to one true component varying in $k$ from position to position, whereas other two components of $\mathbf{x}_{i}$ are set to zero. Estimating the missing color components of $\mathbf{x}(i)$ constitutes the interpolated RGB image $\mathbf{y}(i): Z^{2} \rightarrow Z^{3}$.

This process relates to a sliding supporting window $W=$ $\left\{\mathbf{x}_{i} \in Z^{2} ; i=0,1, \ldots, N-1\right\}$ of finite size $N$, considered with the sample under consideration, sample $\mathbf{x}_{0}$, placed in the center of the window (Fig.2a). The procedure replaces the center $\mathbf{x}_{0}$ by some function of the local neighborhood area $\left\{\mathbf{x}_{1}, \mathbf{x}_{2}, \ldots, \mathbf{x}_{N-1}\right\}$ at a time. The rationale of this approach is to minimize the local distortion and ensure the stationarity of the processes generating the image. 


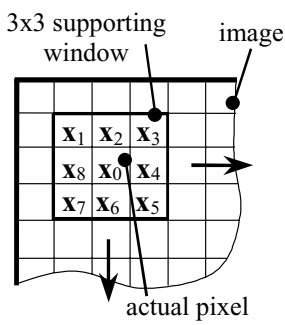

(a)

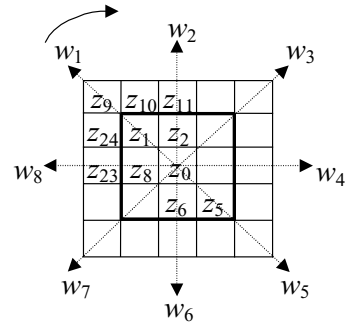

(b)
Fig. 2. Utilized components: (a) sliding supporting window, (b) edge-sensing weighting coefficients.

\section{PROPOSED METHOD}

To follow structural information and interpolate missing image components in sharp shapes, efficient interpolation algorithms incorporate edge information into the interpolation process. The proposed method utilizes the edge-sensing mechanism of [5]:

$$
\begin{gathered}
w_{1}=\frac{1}{1+\left(\left|z_{0}-z_{9}\right|+\left|z_{1}-z_{5}\right|\right) /(2 \sqrt{2})} \\
w_{2}=\frac{1}{1+\left(\left|z_{0}-z_{11}\right|+\left|z_{2}-z_{6}\right|\right) / 2}
\end{gathered}
$$

where $w_{1}$ and $w_{2}$ denote weights in north-west and north directions and $z_{0}, z_{1}, \ldots, z_{24}$ are original gray-scale values of $z(i)$ (Fig.2b). Coefficients $w_{3}, w_{5}, w_{7}$ and $w_{4}, w_{6}, w_{8}$ are calculated applying appropriately the concept of (2) and (3), respectively.

Using eight weighting coefficients, the $\mathrm{G}$ channel is interpolated as follows:

$$
\bar{y}_{02}= \begin{cases}x_{02} & \text { if } z_{0} \cong x_{02} \\ \sum_{i=1}^{N-1} w_{i}^{\prime} x_{i 2}^{\prime} & \text { otherwise }\end{cases}
$$

where $N=9$ relates to a $3 \times 3$ sliding window, $z_{0}$ is the acquired pixel before the transformation (1) in the same spatial position as the color (RGB) vector $\mathbf{x}_{i}$, operator $\cong$ denotes a one to one relationship and $w_{i}^{\prime}=w_{i} / \sum_{j=1}^{N-1} w_{j}$ is the normalized weighting coefficient corresponding to predetermined $\mathrm{G}$ values $x_{i 2}^{\prime}$. For illustration purposes, quantities $x_{(1) 2}^{\prime}$ and $x_{(2) 2}^{\prime}$ are given by:

$$
\begin{gathered}
x_{(1) 2}^{\prime}=\left(x_{(2) 2}+x_{(8) 2}\right) / 2+ \\
\left(\left(z_{1}-z_{5}\right) /(2 \sqrt{2})+\left(z_{11}-z_{0}+z_{23}-z_{0}\right) / 4\right) / 2 \\
x_{(2) 2}^{\prime}=x_{(2) 2}+\left(z_{11}-z_{0}+z_{2}-z_{6}\right) / 4
\end{gathered}
$$

Using the color-difference model of [1] and the $G$ values obtained in (4), the R,B channels are estimated as follows:

$$
\bar{y}_{0 k}= \begin{cases}x_{0 k} & \text { if } z_{0} \cong x_{0 k} \\ \bar{y}_{02}+f_{(2 i) k}^{\prime \prime} & \text { if } z_{0} \cong x_{02} \\ \bar{y}_{02}+f_{(2 i-1) k}^{\prime \prime \prime} & \text { if } z_{0} \cong x_{0(k \pm 2)}\end{cases}
$$

where $k=1$ and $k=3$ characterize the $\mathrm{R}$ and $\mathrm{B}$ channels, respectively; $f_{(2 i) k}^{\prime \prime}=\sum_{i=1}^{(N-1) / 2} w_{i}^{\prime \prime}\left(x_{(2 i) k}-\bar{y}_{(2 i) 2}\right)$ and $f_{(2 i-1) k}^{\prime \prime \prime}=\sum_{i=1}^{(N-1) / 2} w_{i}^{\prime \prime \prime}\left(x_{(2 i-1) k}-\bar{y}_{(2 i-1) 2}\right)$ are defined using normalized weights $w_{i}^{\prime \prime}=w_{2 i} / \sum_{j=1}^{(N-1) / 2} w_{2 j}$ corresponding to edges in north, east, south and west directions; and coefficients $w_{i}^{\prime \prime \prime}=w_{(2 i-1)} / \sum_{j=1}^{(N-1) / 2} w_{(2 j-1)}$ relate to diagonally positioned edges.

Introducing a correction mechanism into the interpolation process improves contrast and accuracy of the initially interpolated G channel. Since the difference plane model of (7) is simple and efficient, it is also perfectly suited to a correction stage. Thus, $\mathrm{G}$ values are corrected as follows:

$$
y_{02}= \begin{cases}\bar{y}_{0 k}+g_{(2 i) k}^{\prime \prime} & \text { if } z_{0} \cong x_{0 k} \\ \bar{y}_{02} & \text { otherwise }\end{cases}
$$

where $g_{(2 i) k}^{\prime \prime}=\sum_{i=1}^{(N-1) / 2} w_{i}^{\prime \prime}\left(\bar{y}_{(2 i) 2}-\bar{y}_{(2 i) k}\right)$ is defined using the weighting coefficients $w_{i}^{\prime \prime}$ of (7).

Considering the corrected $\mathrm{G}$ values of (8) the R,B update is completed using the proposed approach as follows:

$$
y_{0 k}= \begin{cases}y_{0 k} & \text { if } z_{0} \cong x_{0 k} \\ y_{02}+h_{(2 i) k}^{\prime \prime} & \text { if } z_{0} \cong x_{02} \\ y_{02}+h_{(2 i-1) k}^{\prime \prime \prime} & \text { if } z_{0} \cong x_{0(k \pm 2)}\end{cases}
$$

where $h_{(2 i) k}^{\prime \prime}=\sum_{i=1}^{(N-1) / 2} w_{i}^{\prime \prime}\left(\bar{y}_{(2 i) k}-y_{(2 i) 2}\right), h_{(2 i-1) k}^{\prime \prime \prime}=$ $\sum_{i=1}^{(N-1) / 2} w_{i}^{\prime \prime \prime}\left(\bar{y}_{(2 i-1) k}-y_{(2 i-1) 2}\right)$ are defined using the weighting coefficients of (7).

In the case of high correlation between the RGB channels, correction steps of (8) and (9) improve the color appearance of restored images. However, the color difference model can fail due to inadequate shifts in color caused by differences $\left(\bar{y}_{(2 i) 2}-\bar{y}_{(2 i) k}\right),\left(\bar{y}_{(2 i) k}-y_{(2 i) 2}\right)$ and $\left(\bar{y}_{(2 i-1) k}-\right.$ $\left.y_{(2 i-1) 2}\right)$. This can occur due to low correlation between the RGB channels and in the same time the correction process will decrease the image quality in terms of subjective and objective evaluation. Therefore it is reasonable to introduce a global switching control between the non-correction and correction interpolation mode.

Because there is no reason to assume that low correlation between the $R, G$ channels automatically denotes a similar relationship between the G,B channels, two different tests (for $k=1$ and $k=3$ ) are necessary. The tests utilize the correlation coefficients defined as follows:

$$
C_{k}=\frac{\sum\left(\tilde{x}_{i k}-\hat{x}_{k}\right)\left(\tilde{y}_{i 2}-\hat{y}_{2}\right)}{\sqrt{\sum\left(\tilde{x}_{i k}-\hat{x}_{k}\right)^{2}} \sqrt{\sum\left(\tilde{y}_{i 2}-\hat{y}_{2}\right)^{2}}}
$$



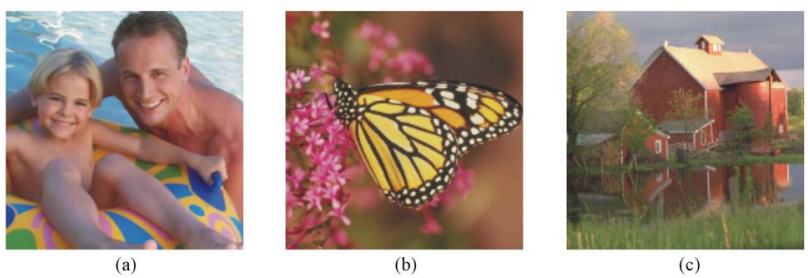

Fig. 3. Test color images: (a) Water, (b) Butterfly, (c) Cottage.

Table 1. Comparison of the presented algorithms using the test image Water.

\begin{tabular}{crrrc}
\hline Method & $M S E_{R}$ & $M S E_{G}$ & $M S E_{B}$ & $N C D$ \\
\hline API & 8.840 & 2.310 & 8.796 & 0.0193 \\
BI & 13.239 & 3.568 & 12.349 & 0.0194 \\
BD & 11.380 & 3.501 & 13.329 & 0.0212 \\
MFI & 8.317 & 2.767 & 7.733 & 0.0189 \\
Proposed & 6.401 & 1.635 & 6.834 & 0.0177 \\
\hline
\end{tabular}

where $i$, for $z_{i} \cong x_{i k}$, characterizes the spatial position of the samples corresponding to the original position of R,B values in the Bayer pattern, $\hat{x}_{k}$ denotes the sample mean related to the $k$-th channel values of $\tilde{\mathbf{x}}(i)$ and $\hat{y}_{2}$ is the sample mean related to the $\mathrm{G}$ channel of $\overline{\mathbf{y}}(i)$ defined, for $\forall\left(z_{(\cdot)} \cong\right.$ $\left.x_{(\cdot) k}\right)$, as follows:

$$
\begin{gathered}
\tilde{x}_{i k}=f_{1,-2,1}\left(x_{(i-1) k}, x_{i k}, x_{(i+1) k}\right) \\
\tilde{y}_{i 2}=f_{1,-2,1}\left(\bar{y}_{(i-1) 2}, \bar{y}_{i 2}, \bar{y}_{(i+1) 2}\right)
\end{gathered}
$$

where $f_{1,-2,1}(\cdot)$ denotes a 1-D high pass filtering with the coefficients $(1,-2,1)$ applied to horizontal and vertical directions, $k$ characterizes the $\mathrm{R}$ and $\mathrm{B}$ channels.

If $C_{k}$ is larger than a chosen threshold $\gamma$, then (9) carries out for the appropriate color channel $k$. Experimentation with a large number of images showed that $\gamma=0.450$ relates to a sufficient performance of the method.

\section{EXPERIMENTAL RESULTS}

To examine the performance of the proposed framework and facilitate comparisons with the state of-the-art CFA interpolation schemes, some widely used natural test color images shown in Fig.3 are utilized. All test images have been normalized to a standard size of $512 \times 512$ pixels with a 8-bit per channel RGB representation.

The proposed method is compared with other CFA interpolation methods, such as adaptive color plane interpolation (API) [4], bilinear interpolation (BI) [6], bilinear difference interpolation (BD) [7] and median filter interpolation (MFI)
Table 2. Comparison of the presented algorithms using the test image Butterfly.

\begin{tabular}{crrrc}
\hline Method & $M S E_{R}$ & $M S E_{G}$ & $M S E_{B}$ & $N C D$ \\
\hline API & 12.006 & 7.056 & 11.344 & 0.0264 \\
BI & 74.045 & 30.738 & 73.374 & 0.0435 \\
BD & 12.646 & 7.522 & 12.439 & 0.0278 \\
MFI & 16.009 & 5.030 & 15.766 & 0.0272 \\
Proposed & 9.677 & 3.678 & 9.597 & 0.0241 \\
\hline
\end{tabular}

Table 3. Comparison of the presented algorithms using the test image Cottage.

\begin{tabular}{ccrcc}
\hline Method & $M S E_{R}$ & $M S E_{G}$ & $M S E_{B}$ & $N C D$ \\
\hline API & 18.728 & 12.800 & 21.709 & 0.0346 \\
BI & 79.839 & 36.644 & 94.344 & 0.0579 \\
BD & 16.901 & 11.646 & 23.168 & 0.0329 \\
MFI & 24.242 & 8.917 & 32.309 & 0.0357 \\
Proposed & 12.444 & 6.801 & 15.275 & 0.0288 \\
\hline
\end{tabular}

[3]. To measure similarity between the original, full RGB, image $\mathbf{o}(i)$ and interpolated image $\mathbf{y}(i)$, we will make use of the mean square error (MSE) and the normalized color difference (NCD) criterion of [8].

Tables 1-3 summarize results corresponding to the restoration of test images shown in Fig.3. Using natural test images we are able to compare performance of the methods in realistic applications, since in these images the correlation between the color channels vary significantly. It can be seen that the flexible design characteristics of the proposed method result in excellent performance as the reported error values indicate.

Figs.4-6 present zoomed parts of the restored images. These images allow for the comparison of the results corresponding to the fundamental BI and currently used API technique with those obtained through the proposed method in terms of a subjective (user-centered) evaluation. It is evident that the BI scheme fails near edges and produces color artifacts. The BD and API schemes produces more quality results. It can be seen that the proposed method is capable of restoring the color images with a high visual quality and avoids color shifts and visual artifacts.

\section{CONCLUSIONS}

A new CFA interpolation approach for single-sensor digital cameras was provided. The proposed edge-sensing method utilizes interpolation and correction steps based on a refined color difference model. Excellent design characteristics of 


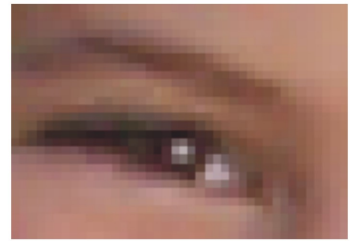

(a)

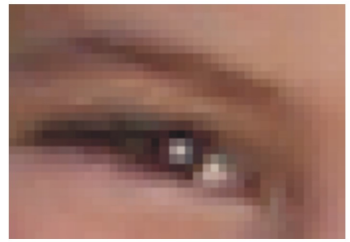

(c)

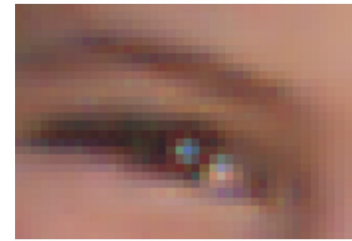

(b)

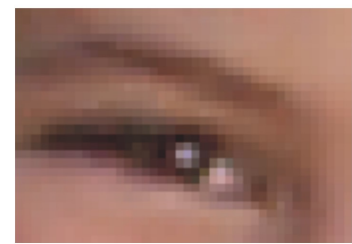

(d)

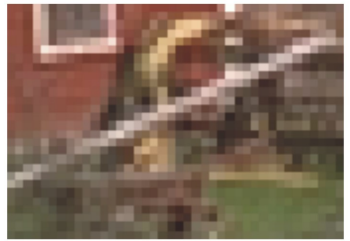

(a)

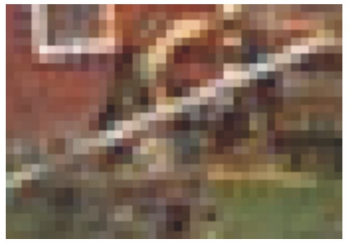

(c)

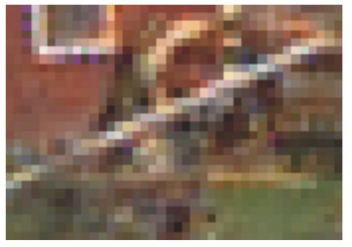

(e)

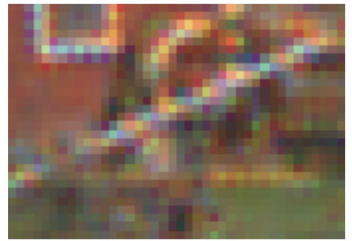

(b)

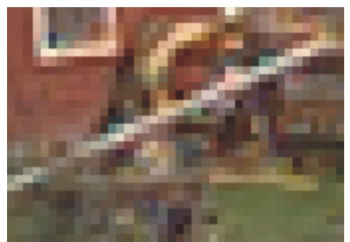

(d)

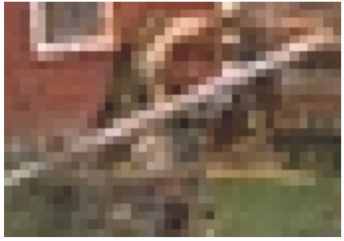

(f)
Fig. 4. Zoomed parts of the test image Water: (a) original image, (b) BI, (c) API, (d) proposed method.

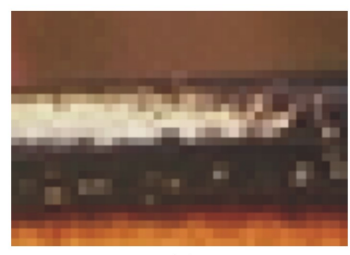

(a)

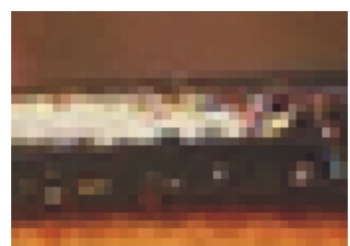

(c)

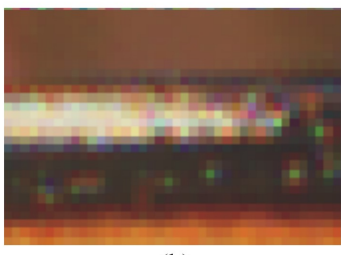

(b)

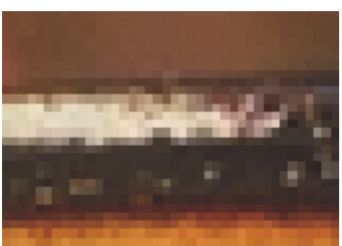

(d)
Fig. 5. Zoomed parts of the test image Butterfly: (a) original image, (b) BI, (c) API, (d) proposed method.

the proposed method result in interpolated images which are sharp, naturally colored and attractive for viewing. Moreover, the new method outperforms previously developed interpolation methods in terms of commonly used objective criteria as well as the subjective evaluation.

Since the introduced corrections step control can be realized depending on the local image statistics, future research will focus on a locally adaptive switching between interpolation and correction steps. This could bring another improvement in the restored image quality.

\section{REFERENCES}

[1] J. Adams, "Design of practical color filter array interpolation algorithms for digital cameras," Proc. of the SPIE, vol. 3028, pp. 117-125, February 1997.
Fig. 6. Zoomed parts of the test image Cottage: (a) original image, (b) BI, (c) BD, (d) API, (e) MFI, (f) proposed method.

[2] B.E. Bayer, "Color imaging array," U.S. Patent 3971 065, 1976.

[3] W.T. Freeman, "Median filter for reconstructing missing color samples," U.S. Patent 5373 322, 1988.

[4] J.F. Hamilton and J.E. Adams, "Adaptive color plane interpolation in single sensor color electronic camera," U.S. Patent 5 629 734, 1997.

[5] N. Kehtarnavaz, H.J Oh, and Y. Yoo, "Color filter array interpolation using color correlations and directional derivatives," Journal of Electronic Imaging, 2003.

[6] P. Longere, Z. Xuemei, P.B. Delahunt, and D.H. Brainard, "Perceptual assessment of demosaicing algorithm performance," Proceedings of the IEEE, vol. 90, pp. 123-132, January 2002.

[7] S.C. Pei and I.K. Tam, "Effective color interpolation in CCD color filter arrays using signal correlation," IEEE Trans. Circuits and Systems for Video Technology, vol. 13, pp. 503-513, June 2003.

[8] K.N. Plataniotis and A.N. Venetsanopoulos, Color Image Processing and Applications. Springer Verlag, 2000.

[9] G. Sharma, Digital Color Imaging Handbook. CRC Press, Boca Raton, 2002. 